\title{
Expression Grade Sirtuin-1 (SIRT-1) in Tumor Tissue in Women with Breast Cancer: A New Biomarker
}

\section{Prognosis?}

\author{
Juliano Sartori ${ }^{1}$ and Rodolfo Herberto Schneider ${ }^{2}$ \\ 1. Centro de Oncologia Clínica e Radioterapia Erechim, Erechim 99701-418, Brazil \\ 2. Pontificia Universidade Católica do Rio Grande do Sul (PUCRS), Porto Alegre 90619-900, Brazil
}

\begin{abstract}
Breast cancer is a complex and heterogeneous disease with different clinical outcomes. The investigations of new biomolecular markers are essential to know the prognosis and improve the clinical management of patients. The SIRT-1 (sirtuin-1) is a histone deacetylase implicated in various epigenetic critical functions for the cells and the maintenance of genomic stability. The objective of this study is to investigate the grade of expression of the SIRT-1 (sirtuin-1) and the prognostic value in overall survival of women with breast cancer. Retrospective cohort of 457 women with breast cancer has been researched, undergoing treatment in Erechim-RS from 2003 to 2013 and followed until July 2015. The degree of SIRT-1 expression was investigated by immunohistochemistry in 123 patients $(26.9 \%)$ of the cohort. The OS (overall survival) from specific disease and risk of death from breast cancer were estimated by Kaplan-Meier and Cox's proportional risks. The median age of 57.4 years cohort with OS of $79.6 \%$ in 5 years and $69.1 \%$ at 10 years, with follow-up time of 61.9 months are revealed in this work. The SIRT-1 overexpression was found in $6.5 \%$ of cases and characterized a subgroup of women with shorter survival and increased risk of death from breast cancer $(H R=2.66$; 95\% CI 1.03 to $6.86 ; p=0.043)$ and adjusted by age $(H R=2.86 ; 95 \%$ CI 1.11 to $7.38 ; p=0.030)$, histology $(H R=2.79 ; 95 \%$ CI 1.07 to $7.28 ; p=0.036)$, lymph nodes $(H R=2.73 ; 95 \%$ CI 1.06 to $7.04 ; p=0.037)$, Her-2 (HR=2.82; 95\% CI 1.07 to $7.44 ; p=0.036)$; chemotherapy $(H R=2.90 ; 95 \%$ CI 1.11 to $7.60 ; p=0.030)$ and radiotherapy $(H R=2.71 ; 95 \%$ CI 1.05 to $7.01 ; p=0.040)$. In regressive multivariate models adjusted for age, status of axillary lymph nodes, Her-2 expression and proliferation index (Ki-67), the grade of expression of the SIRT-1 maintained association with poor prognosis. From the study, it can be concluded that the assessment of the SIRT-1 expression is an independent prognostic biomarker in breast cancer.
\end{abstract}

Key words: Breast cancer, sirtuin-1, survival, carcinogenesis, repression epigenetics, prognosis, immunohistochemistry.

\section{Introduction}

Breast cancer is a progressive disease with heterogeneous clinical and biological characteristics that is a relevant problem of global public health, because it has high rates of incidence, morbidity and mortality [1-3]. Epidemiologically, breast cancer is the most common malignancy in Europe and the United States and the second leading cause of cancer related death in women [4]. In Europe were estimated 464,000 new cases of breast cancer with 131,000 deaths from the disease in 2012 and in the United States for the year

Corresponding author: Juliano Sartori, M.D., doctor in biomedical gerontology, research fields: oncology and human phisiology.
2016, it is estimated about 246,660 new cases of breast cancer in women and 40,450 deaths from the disease [2, 4]. In Brazil, for the year 2016, were estimated 596,000 new cases of cancer, from these, 57,960 cases are of breast cancer, according to a report published by the National Cancer Institute [5]. For the Southern region of Brazil, it was estimated 10,970 new cases of breast cancer for the year 2016, accounting for $25 \%$ of all cases of cancer estimated for the women of this region [5].

The prognosis and treatment of breast cancer are based on several biological factors, such as age, tumor size, metastases in the axillary lymph nodes, systemic staging, histological grade, expression profile of 
hormone receptors, expression profile receptor human epidermal growth factor (Her-2) and proliferation index (Ki-67) that have genetic signatures themselves to this disease [6-9]. Thus, breast cancer constitutes a set of disorders with distinct molecular portraits and needs for its approach, biomedical research to understand the biological and molecular processes involved in their oncogenesis, evolution and mechanisms of resistance of tumor cells and relationship with clinical outcomes $[1,2,10]$.

The complexity of breast oncogenesis, epigenetic factors, plays an important role because they are molecular events involved in various physiological activities of cells and is essential for the regulation of gene expression [11]. Several molecular mechanisms involved in oncogenesis are unknown, but the changes in the acetylation and deacetylation of histones, particularly in the sirtuin family of molecules (Sir2), have been studied and related to carcinogenesis [12-14].

Sirtuins are intracellular protein molecules, Class III of histone deacetylases and they were discovered in Saccharomyces cerevisiae yeast $[12,15]$. In humans, they are a group of proteins composed of seven members with different metabolic functions, essential for the maintenance of cellular homeostasis and directly related to silencing cellular process $[13,16,17]$.

The SIRT-1 (sirtuin-1) is the main molecule described in the sirtuin family $[12,17]$ and has been associated with silencing of genes, cell cycle control, apoptosis, the energy homeostasis and aging [18]. This molecule acts in many vital biological processes of cell gene expression, and consequently, its overexpression may be related to the biological behavior of breast cancer destabilization of the regulation of estrogen receptors or using other substrates, such as p53 [19].

The objective of this research was to investigate the grade of SIRT-1 expression in biopsies of tumor tissue of women with breast cancer and its prognostic value in overall survival specific disease.

\section{Methods}

\subsection{Study Design and Population}

Research with retrospective design, based on hospital medical records, for composition of a cohort of 457 female patients with breast cancer, diagnosed at the Pathology Laboratory (Medical Laboratory Diagnostics) Erechim-RS and undergoing cancer treatment in the period of 2003 2013 in the following Erechim-RS institutions: Erechim Clinical Oncology Center (COC), Erechim Santa Terezinha Hospital (FHSTE) and Erechim Charity Hospital (HCE). The recruitment period for composition of the cohort was the first day of 2003 (date of initial entry into the cohort) and on December 31, 2013 (end date of entry into the cohort). The inclusion criteria for the composition of the study cohort were diagnosed with breast cancer in women, confirmed by pathological examination, and the completion of cancer treatment from 2003 to 2013. The first 123 consecutive cases which entered the cohort were selected from the first of January 2003, to research the grade of expression of the SIRT-1 (sirtuin-1) by immuno histochemical technique.

Information on the initial exposure (date of histological diagnosis) and the outcome (death) were obtained by analyzing the medical records. The final follow-up date of patients in the cohort, to analyze the outcome (death) was July 31, 2015. On that date, all patients who were alive were censored. The variables studied or prognostic factors were: age at diagnosis; histological type; nuclear histological grade (low, intermediate and high); status of the axillary lymph nodes (profile of metastatic involvement of axillary lymph nodes); hormone receptors (expression of estrogen and progesterone receptors); cell proliferation Ki-67 (low and high grade); grade of expression of human epidermal growth factor receptor (Her-2); TNM staging (AJCC): I to IV; type of treatment used (surgery, radiotherapy, chemotherapy, hormone therapy, anti-Her-2 targeted therapy with Herceptin $\AA$ ) and grade of expression of SIRT-1 (sirtuin-1). 


\subsection{Technique for SIRT-1 Expression Level Research}

The grade of SIRT-1 expression was investigated by immunohistochemical technique and the following steps were taken: fixing and obtaining histological sections from the paraffin block with signalized blade Dako, submitted to the technique of immunoperoxidase antigen retrieval performed in Dako instrument, PT Link, with three processing in an integrated, comprising the deparafinization, rehydration and antigen retrieval with FLEX caps (Dako) low or high $\mathrm{pH}$; Incubation with primary antibodies for $20 \mathrm{~min}$ at room temperature; Use of IgG antibody, SIRT-1 human recombinant enzyme (No. 50081-Fluorogenic SIRT1) of Bioscience Laboratory (BPS); Amplification was performed with En Vision FLEX (Dako) systems with secondary antibodies conjugated to polymer En Vision and developed with chromogenic system DAB + liquid FLEX (Dako); automated processing platform Dako Autostainer Link 48; conducting internal and external positive controls to test the fidelity of reactions; Mounting of blades in Coversliper Dako with Dako cover slips; analysis by the pathologist Sector of Immunohistochemistry of Diagnostic Medicine Laboratory by optical microscopy with fluorescence reader.

The criteria used to analyze the grade of expression of the SIRT-1 (sirtuin-1) were as follows.

The percentage level of expression of SIRT-1 determined by the pathologist was recorded as a numeric variable from zero to $100 \%$, according to immunofluorescence reaction SIRT-1 in the nucleus of tumor cells by light microscopy field.

According to the level of expression of SIRT1 obtained in the investigation the patients were classified as:

- Negative expression (0): grade of nuclear expression of SIRT-1 absent or up to $1 \%$ of tumor cells;

- Low expression $(1+)$ : grade of nuclear expression of SIRT-1 between $1 \%$ and $49 \%$ of the tumor cells (Fig. 1);
- Moderate expression (2+): grade of nuclear expression of SIRT1 between $50 \%$ and $79 \%$ of the tumor cells (Fig. 2);

- Overexpression (3+): grade of nuclear expression of SIRT1 above $80 \%$ of the tumor cells (Fig. 3 ).

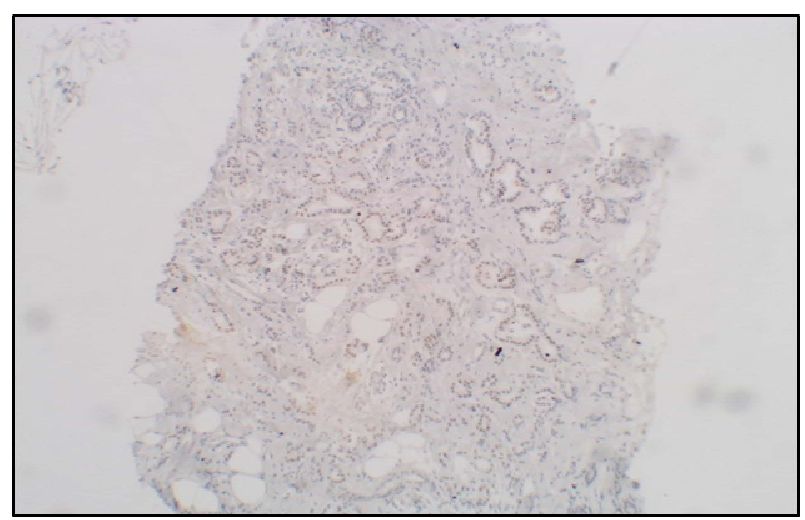

(a)

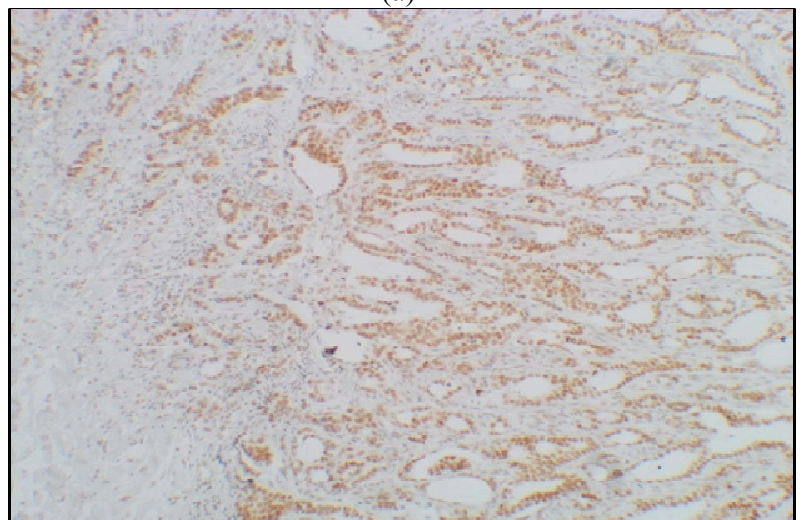

(b)

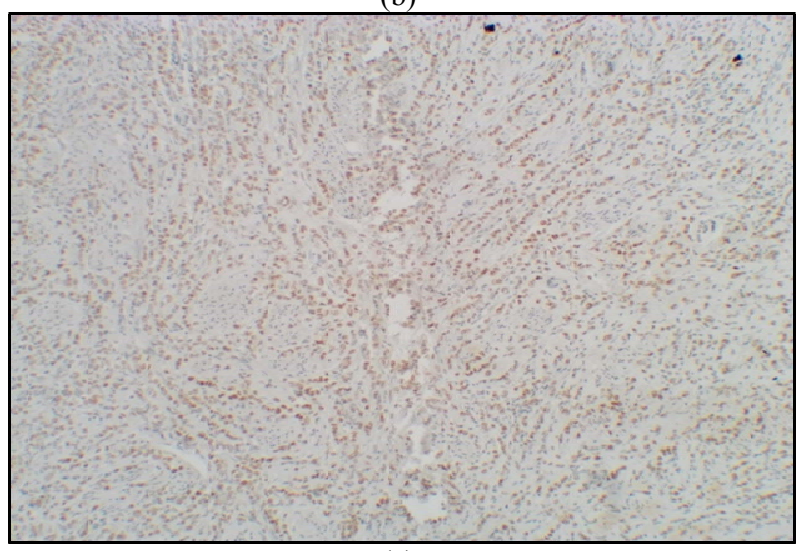

(c)

Fig. 1 Microscopic image of tumor tissue of breast cancer in female patients with: (a) low expression of SIRT-1 (grade of $40 \%$ expression); (b) moderate expression of SIRT-1 (grade of 70\% expression); (c) overexpression expression of SIRT-1 (grade of $80 \%$ expression).

Source: Immunohistochemical technique, increased 100x, the author, 2016. 


\subsection{Statistical Analysis}

The results were statistically analyzed using SPSS Version 21.0. A descriptive analysis of all prognostic variables surveyed in Table 1 with a breakdown of its characteristics and frequencies found was presented.

The survival probabilities each time were estimated using the Kaplan-Meier method and the comparison between the curves was held by the log-rank test. In order to estimate the effects of each variable on survival of patients were created proportional hazards models Cox [20]. Survival curves decomposed according to the variables in the model of the univariate analysis were presented and the relative risks (HR (hazard ratio)) estimated. The confidence intervals were $95 \%$.

The variables that were statistically significant in the univariate analysis and relevance in the pathogenesis of breast cancer were tested in a multivariate model, by the method of Cox proportional hazards. In the final multivariate analysis were calculated the HR for each proposed model for investigate possible predictive prognostic associations according to the purpose of the study [20].
The study was approved by the Research Ethics Committee of PUCRS as reported number 465.362 of November 22, 2013 were adopted ethical research recommendations of Resolution 466/12 of the National Health Council of the Ministry of Health of Brazil.

\section{Results}

The cohort median follow-up time was 61.93 months, with a minimum follow-up time was 1.37 months (42 days) and the maximum follow-up time was 153.03 months (12.75 years). At the end of cohort follow-up, 364 patients $(79.6 \%)$ were alive and were censored on July 31, 2015, and 93 patients (20.4\%) died due to breast cancer.

The median age of the study population was 57.48 $(\mathrm{SD}) \pm 27.76$ years, and the minimum age was 25 years and the maximum of 92 years.

The characteristics of the population according to the investigated variables are shown in Table 1.

The median OS (overall survival) specific disease obtained at the end of the study, the Kaplan-Meier method was 120.243 months (10.02 years) with a standard deviation of 2.928 (CI 95\% 114.504 125.982) months. The survival median estimates calculated for

Table 1 Description and frequency of variables searched for composition of the total cohort and SIRT-1 cohort.

\begin{tabular}{|c|c|c|c|c|c|c|}
\hline \multirow{2}{*}{ Variables } & & \multicolumn{2}{|c|}{ Cohort total } & \multicolumn{2}{|c|}{ Cohort SIRT-1 } & \multirow{2}{*}{$p^{1}$} \\
\hline & & $n=457$ & Percentage (\%) & $n=123$ & Percentage (\%) & \\
\hline \multirow{4}{*}{ Age range } & $<50$ years old & 149 & 32.6 & 48 & 39.0 & 0.045 \\
\hline & $50 \sim 59$ & 115 & 25.2 & 28 & 22.8 & \\
\hline & $60 \sim 69$ & 90 & 19.7 & 26 & 21.1 & \\
\hline & $\geq 70$ & 103 & 22.5 & 21 & 17.1 & \\
\hline \multirow{3}{*}{ Histology } & Invasive ductal & 396 & 86.9 & 105 & 85.4 & 0.609 \\
\hline & Invasive lobular & 48 & 10.5 & 16 & 13.0 & \\
\hline & Mucinous & 12 & 2.6 & 2 & 1.6 & \\
\hline \multirow{4}{*}{ Tumor size } & $\mathrm{T} 1(<2 \mathrm{~cm})$ & 131 & 28.7 & 30 & 24.4 & 0.103 \\
\hline & $\mathrm{T} 2(2 \sim 4.9 \mathrm{~cm})$ & 265 & 58.0 & 71 & 57.7 & \\
\hline & $\mathrm{T} 3(\geq 5 \mathrm{~cm})$ & 34 & 7.4 & 17 & 13.8 & \\
\hline & T4 (inflammatory) & 27 & 5.9 & 5 & 4.1 & \\
\hline \multirow{4}{*}{ Axillary lymph nodes } & N0 (negatives) & 225 & 49.2 & 48 & 39.0 & 0.008 \\
\hline & N1(<4 positive lymph nodes) & 146 & 31.9 & 45 & 36.6 & \\
\hline & $\mathrm{N} 2(\geq 4$ positive lymph nodes) & 71 & 15.5 & 27 & 22.0 & \\
\hline & N3 (positive lymph nodes fused) & 15 & 3.3 & 3 & 2.4 & \\
\hline \multirow{2}{*}{ Metastasis } & Without & 452 & 98.9 & 122 & 88 & 0.569 \\
\hline & With & 5 & 1.1 & 1 & 5 & \\
\hline
\end{tabular}


(Table 1 continued)

\begin{tabular}{|c|c|c|c|c|c|c|}
\hline \multirow[t]{2}{*}{ Variables } & & \multicolumn{2}{|c|}{ Cohort total } & \multicolumn{2}{|c|}{ Cohort SIRT-1 } & \multirow[t]{2}{*}{$P^{1}$} \\
\hline & & $n=457$ & Percentage $(\%)$ & $n=123$ & Percentage $(\%)$ & \\
\hline \multirow[t]{4}{*}{ Staging (TNM) } & I & 91 & 19.9 & 17 & 13.8 & \multirow[t]{4}{*}{0.032} \\
\hline & II & 249 & 54.5 & 68 & 55.3 & \\
\hline & III & 112 & 24.5 & 37 & 30.1 & \\
\hline & IV & 5 & 1.1 & 1 & 0.08 & \\
\hline \multirow[t]{3}{*}{ Histologic grade } & Low & 41 & 9.0 & 5 & 4.1 & \multirow[t]{3}{*}{0.111} \\
\hline & Intermediate & 301 & 65.8 & 84 & 68.3 & \\
\hline & High & 115 & 25.2 & 34 & 27.6 & \\
\hline \multirow[t]{2}{*}{ Estrogen receptor(ER) } & Negative & 92 & 20.1 & 27 & 22.0 & \multirow[t]{2}{*}{0.556} \\
\hline & Positive & 365 & 79.9 & 96 & 78.0 & \\
\hline \multirow{2}{*}{$\begin{array}{l}\text { Progesterone } \\
\text { receptor(PR) }\end{array}$} & Negative & 118 & 25.8 & 30 & 24.4 & \multirow[t]{2}{*}{0.672} \\
\hline & Positive & 339 & 74.2 & 93 & 75.6 & \\
\hline \multirow[t]{2}{*}{ HER-2 } & Negative & 367 & 80.3 & 93 & 75.6 & \multirow[t]{2}{*}{0.108} \\
\hline & Overexpression & 90 & 19.7 & 30 & 24.4 & \\
\hline \multirow{2}{*}{$\begin{array}{l}\text { Ki-67 } \\
\text { (proliferation index) }\end{array}$} & Low & 132 & 28.9 & 60 & 48.8 & \multirow[t]{2}{*}{0.001} \\
\hline & High & 325 & 71.1 & 63 & 51.2 & \\
\hline \multirow{5}{*}{ Molecular profile } & Luminal A & 111 & 24.3 & 33 & 26.8 & \multirow[t]{5}{*}{0.758} \\
\hline & Luminal B HER-2 negative & 216 & 47.3 & 49 & 39.8 & \\
\hline & Luminal B HER-2 positive & 64 & 14.0 & 22 & 17.9 & \\
\hline & HER-2 Overexpression & 26 & 5.7 & 9 & 7.3 & \\
\hline & Triple negative & 40 & 8.8 & 10 & 8.1 & \\
\hline \multirow{3}{*}{ Surgery } & Not performed & 1 & 0.2 & 1 & 0.8 & \multirow[t]{3}{*}{0.478} \\
\hline & Conservative & 181 & 39.6 & 51 & 41.5 & \\
\hline & Radical & 275 & 60.2 & 71 & 57.7 & \\
\hline \multirow{2}{*}{ Chemotherapy } & Not performed & 117 & 25.6 & 16 & 13.0 & \multirow[t]{2}{*}{0.000} \\
\hline & Performed & 340 & 74.4 & 107 & 87.0 & \\
\hline \multirow{2}{*}{ Radiotherapy } & Not performed & 168 & 36.8 & 29 & 23.6 & \multirow[t]{2}{*}{0.000} \\
\hline & Performed & 289 & 63.2 & 94 & 76.4 & \\
\hline \multirow{2}{*}{ Endocrine therapy } & Not performed & 89 & 19.5 & 23 & 18.7 & \multirow[t]{2}{*}{0.800} \\
\hline & Performed & 368 & 80.5 & 100 & 81.3 & \\
\hline \multirow{5}{*}{$\begin{array}{l}\text { Herceptin }{ }^{\circledR} \\
\text { target) }\end{array}$} & Not performed & 41 & 45,6 & 21 & 70,0 & \multirow[t]{3}{*}{0,154} \\
\hline & Performed & 49 & 54,4 & 9 & 30,0 & \\
\hline & Censored & & & & & \\
\hline & Live & 364 & 79,6 & 87 & 70,7 & 0,004 \\
\hline & Events deaths & 93 & 20,4 & 36 & 29,3 & \\
\hline
\end{tabular}

Source: The author, 2016.

the outcome of death from breast cancer were $96.9 \%$ in 12 months; $92.1 \%$ at 24 months; $88.3 \%$ at 36 months; $83.6 \%$ in 48 months; $79.6 \%$ in 60 months and $69.1 \%$ in 10 years. The survival curve is shown in Fig. 2.

The 123 cases selected for investigation of the grade of expression of the SIRT-1, represented $26.9 \%$ of the total cohort of female patients with breast cancer $(n=457)$.

Table 2 shows the description of the cases classified according to the level of expression of SIRT-1 found from the immunohistochemical research and as criteria used for classification. In the analysis of the degree of expression of the SIRT-1, we observed nine cases (7.3\%) that showed no expression; 71 cases (57.7\%) classified as low expression; 35 cases (28.5\%) classified as moderate expression and eight cases $(6.5 \%)$ classified as overexpression.

According to the main goal in this research were 
A New Biomarker Prognosis?

calculated survival median estimates and risk of death in univariate and multivariate Cox models, to check the impact of the grade of SIRT-1 expression in the prognosis of patients investigated. To model these analyzes, patients were grouped and classified into two groups according to overexpression or not of the SIRT-1.

According to the primary objective in this research were calculated survival median estimates and risk of death in univariate and multivariate Cox models, to check

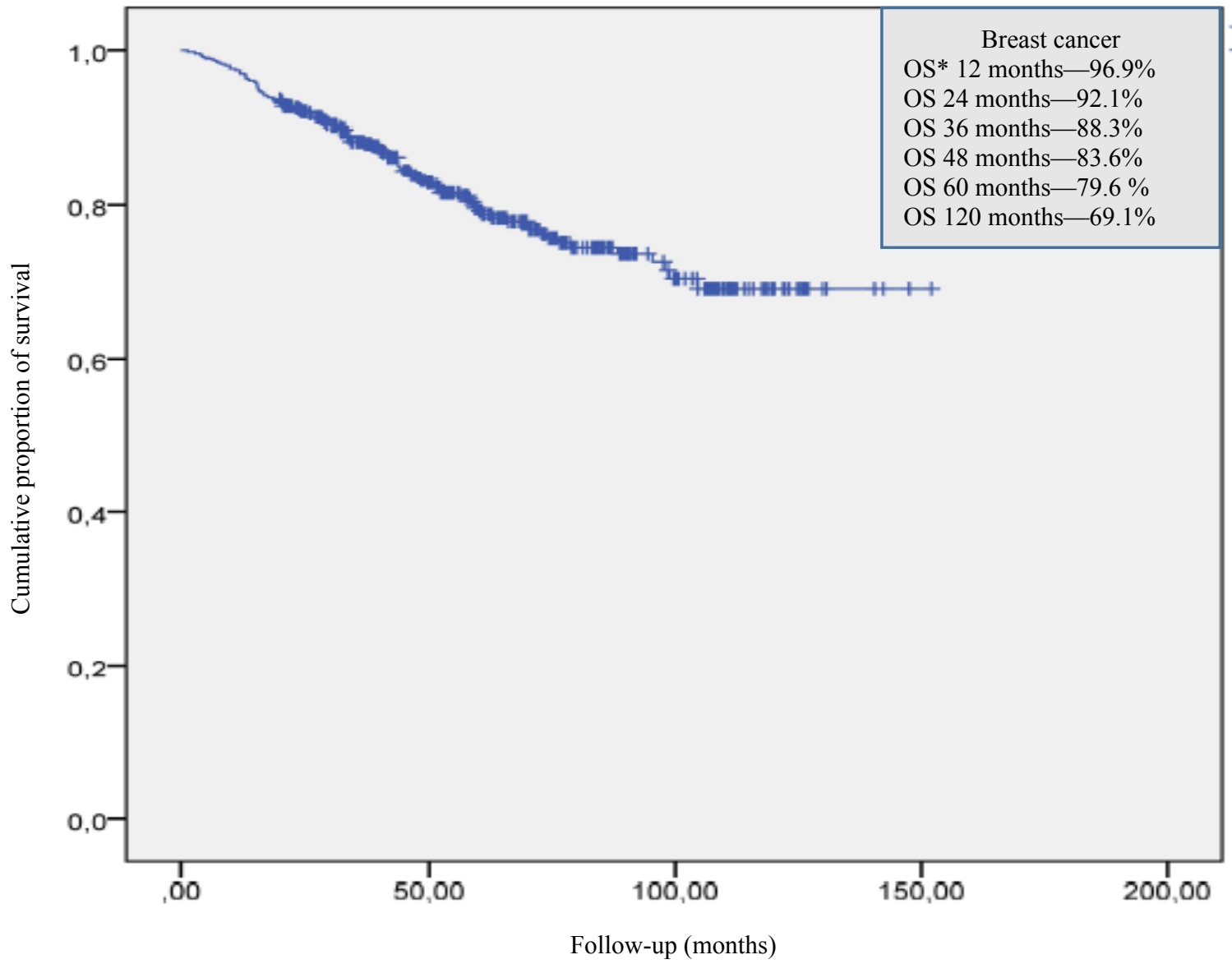

\begin{tabular}{lllllll}
\hline Full cohort & Cases $(n)$ & Deaths $(n)$ & Deaths $(\%)$ & OS* (meses) & CI 95\% & $\begin{array}{l}\text { Kaplan-Meier } \\
\text { Standard deviation: }\end{array}$ \\
\hline Breast cancer & 457 & 93 & 20.4 & 120.243 & $114.504 \sim 125.982$ & 2.928 \\
\hline
\end{tabular}

Fig. 2 Global survival curve specific disease (Kaplan-Meier) in months of patients with breast cancer during the follow-up of the cohort.

*estimated median overall survival specific disease every time;

Source: The author, 2016.

Table 2 Description of the grade of expression of the SIRT-1 (sirtuin-1) in patients with breast cancer and number of events occurred during follow-up.

\begin{tabular}{lcccc}
\hline \multirow{2}{*}{ Grade of expression (SIRT-1) } & Cohort SIRT-1 & \multicolumn{3}{c}{ Events } \\
\cline { 3 - 5 } & $n=123$ & \% (SIRT-1 expression) & $n=35$ & $\%$ (deaths) \\
\hline Negative expression (0) & 9 & 7.3 & 3 & 33.3 \\
Low expression (1+) & 71 & 57.7 & 18 & 25.4 \\
Moderate expression (2+) & 35 & 28.5 & 9 & 25.7 \\
Overexpression (3+) & 8 & 6.5 & 5 & 62.5 \\
\hline
\end{tabular}

Source: The author, 2016. 
the impact of the grade of SIRT-1 expression in the prognosis of patients investigated. To model these analyzes, patients were grouped and classified into two groups according to overexpression or not the SIRT-1.

Thus, the groups were classified as:

- Sirtuin-1 (SIRT-1) negative group: The cases considered the patients with negative expression (0) and low expression $(1+)$ and moderate expression $(2+)$ of SIRT-1;

- Sirtuin-1 (SIRT-1) overexpressed group: We considered the patients with overexpression (3+).

The curves of overall survival specific disease, estimated for 123 patients with breast cancer, compared to the profile of the expression of the sirtuin-1 (SIRT-1) investigated by immunohistochemistry, are shown in Fig. 3.

According to the analysis and criteria used in this study, we observed 115 cases $(93.5 \%)$ with negative expression of sirtuin-1 (SIRT-1), having been 30 deaths in this group, accounting for $26.1 \%$ of the cases. The median overall survival specific disease, estimated for patients with negative expression of sirtuin-1 (SIRT-1), was 115.541 months (CI 95\% 107.324 123.758). The overexpression of sirtuin-1 (SIRT-1) was observed in eight cases $(6.50 \%)$ and five deaths occurred in this group, accounting for $62.5 \%$ of

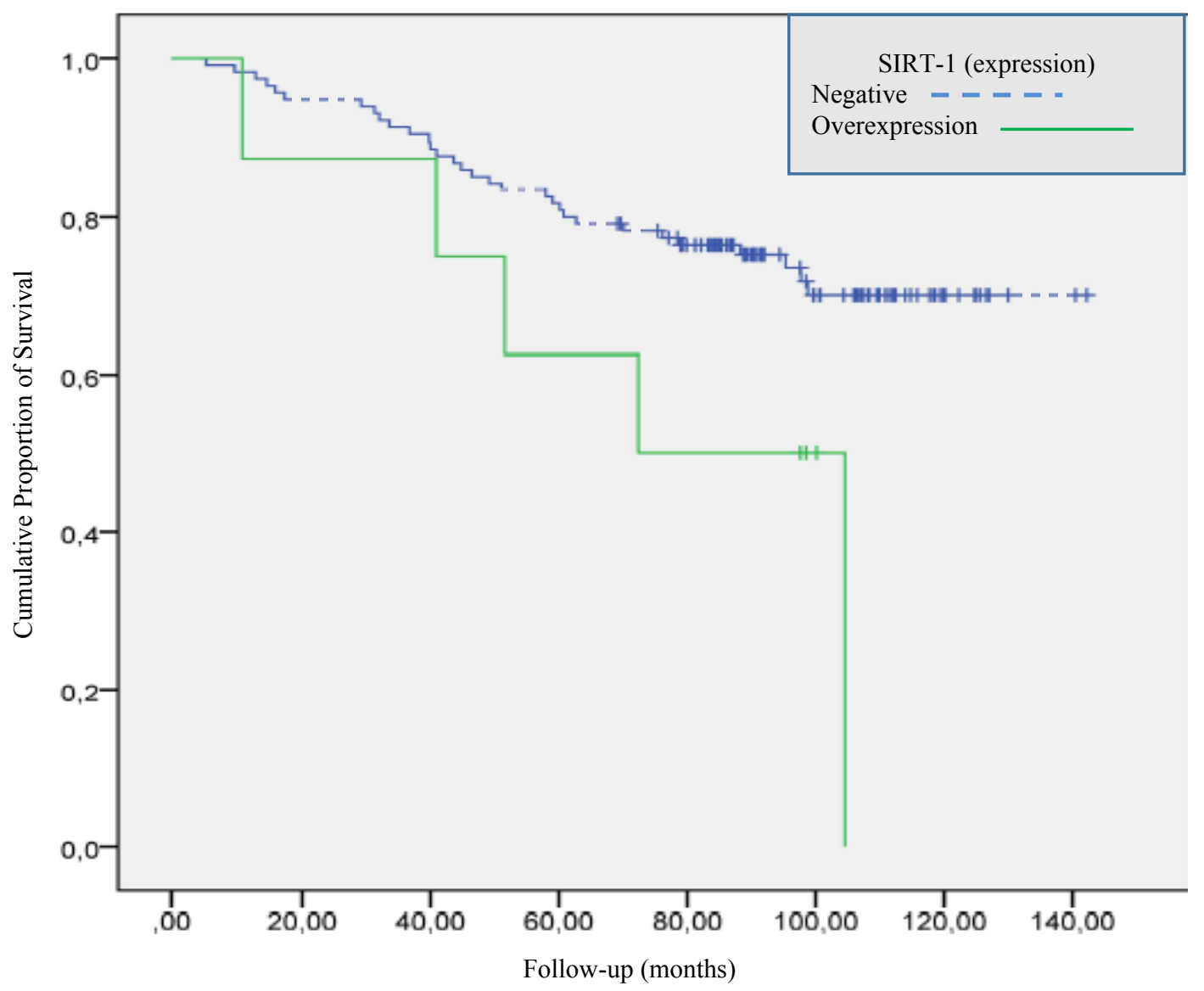

\begin{tabular}{lllllll}
\hline SIRT-1 & Cases (n) & Deaths (n) & Deaths (\%) & OS (meses) & CI 95\% & $\begin{array}{l}\text { Log Rank } \\
X^{2}: 4.452 \\
p=0.035\end{array}$ \\
\hline Negative & 115 & 31 & 26.9 & 115.541 & $107.324 \sim 123.758$ & \\
Overexpression & 8 & 5 & 62.5 & 74.221 & $47.735 \sim 100.707$ & \\
\hline
\end{tabular}

Fig. 3 Overall survival curves specific disease (Kaplan-Meier) months of the cohort of patients with breast cancer according to expression of sirtuin-1 (SIRT-1).

Source: The author, 2016. 
the cases. The median overall survival particular disease, estimated for patients with overexpression sirtuin-1 (SIRT-1), was 74.221 months (CI 95\% 47.735 100.707). The global log-rank test rejected the similarity hypothesis (parallelism) between the survival distributions curves $\left(X^{2}: 4.452 ; p=0.035\right)$. Thus, the survival curves stratified according to the expression profile of sirtuin-1 (SIRT-1) in patients with breast cancer showed a significant difference between them $(p<0.05)$.

The group of patients with overexpression of SIRT-1 accounted for $6.5 \%$ of the samples investigated. The general characteristics of these samples according to the variables studied are described below:

- The median age was 58 years, and the youngest was 35 and the oldest 77 years;

- The histological type identified in all cases was invasive ductal carcinoma;

- The tumor size was reported as T2 $(2 \mathrm{~cm}$ to $4.9 \mathrm{~cm})$ in four cases $(50 \%)$ and $\mathrm{T} 3(>5.0 \mathrm{~cm})$ in four cases $(50 \%)$;

- The metastatic involvement in axillary lymph nodes was shown in four cases $(50 \%)$ and four cases $(50 \%)$ had no metastases in the axillary lymph nodes;

- In the clinical staging (TNM), we identified three patients (37.5\%) with Stage II (limited disease) and five patients (62.5\%) with Stage III (advanced);

- The histological grade was ranked as high in five cases $(62.5 \%)$ and intermediate in three cases $(37.5 \%)$;

- The profile of expression of hormone receptors (estrogen and progesterone) was negative in four cases $(50 \%)$ and positive in four patients $(50 \%)$. There was no specific difference in the individual analysis of estrogen and progesterone receptors;

- The cell proliferation index (Ki-67) was high grade in seven patients $(87.5 \%)$ and identified one case (12.5\%) low grade;

- The Her-2 profile was negative in five cases (62.5\%) and overexpressed in three cases (37.5\%);

- In the classification according to the molecular profiling, we identified one case (12.5\%) categorized as Luminal A, three cases $(37.5 \%)$ categorized as Luminal B Her-2 negative, two cases (25\%) categorized as Her-2 overexpressed and two cases $(25 \%)$ categorized as negative triple;

- In the type of surgical treatment used, we observed that five patients $(62.5 \%)$ were subjected to mastectomy and three patients $(37.5 \%)$ were subjected to conservative surgery;

- Chemotherapy and radiation were employed in all cases;

- The hormone therapy was performed in five patients $(50 \%)$;

- The anti-Her-2 targeted therapy with Herceptin ${ }^{\circledR}$ was done in one case (50\%).

In order to check the prognostic effect of the grade of expression of the sirtuin-1 (SIRT-1), according to the risk of death from breast cancer, the HR (hazard ratio) were calculated for the SIRT-1 adjusted for variables selected. Table 3 shows the description of the variables and the respective HR obtained in each analysis by univariate Cox regression model. The grade of expression of the SIRT-1 had an HR of 2.66 (95\% CI $1.03 \sim 6.86, p=0.043$ ).

The prognostic variables that were significant in the univariate model and which are relevant to the pathogenesis of SIRT-1 were selected for preparation of regressive multivariate models, the Cox model. The selected variables were age, axillary lymph nodes, TNM classification, histologic grade, the Her-2 profile, cell proliferation $\mathrm{Ki}-67$, the profile of hormone receptors and the profile according to the molecular classification. Table 4 shows the description of the models submitted to multivariate analysis of SIRT-1 expression, adjusted according to each group of selected variables.

The SIRT1 presented associated prognosis with increased risk of death from breast cancer, significant and independent, in almost all proposed multivariate models.

\section{Discussion}

Breast cancers are different in their natural history, 
and in different responses to the treatments altogether [21]. There are several phenotypes of tumors that determine a specific molecular picture for each cancer. These phenotypic changes are the result of many signs of transcription that are involved in oncogenesis causing a biological diversity of tumors [21, 22]. Understanding the genetic complexity of breast cancer is an ongoing challenge to researchers.

The discovery of new molecular markers involved in the pathogenesis of breast cancer is fundamental to optimize treatment and determine with greater certainty the prognosis of patients with the disease. In addition to markers already established in clinical practice, as the expression of estrogen receptor, progesterone receptor, Her-2 and Ki-67, it is necessary subcategorize some cancers that exhibit expression of negativity for the known biomarkers and thereby evolve biological behavior and more aggressive clinical outcomes [23].

Table 3 Univariate analysis of SIRT1 adjusted to occurrence of death according to the description of each prognostic variable in the cohort of female patients with breast cancer.

\begin{tabular}{llll}
\hline Adjusted variables & HR* & CI 95\%** & $p^{* * *}$ \\
\hline SIRT-1 unadjusted & 2.66 & $(1.03 \sim 6.86)$ & 0.043 \\
Age & 2.86 & $(1.11 \sim 7.38)$ & 0.030 \\
Histology & 2.79 & $(1.07 \sim 7.28)$ & 0.036 \\
Tumor size & 1.92 & $(0.71 \sim 5.20$ & 0.198 \\
Lymph nodes & 2.73 & $(1.06 \sim 7.04)$ & 0.037 \\
TNM & 2.25 & $(0.87 \sim 5.81)$ & 0.096 \\
Grade & 1.87 & $(0.71 \sim 4.92)$ & 0.206 \\
Her-2 & 2.82 & $(1.07 \sim 7.44)$ & 0.036 \\
Ki-67 & 2.48 & $(0.95 \sim 6.42)$ & 0.062 \\
Estrogen receptor & 2.42 & $(0.89 \sim 6.60)$ & 0.085 \\
Progesterone receptor & 2.13 & $(0.79 \sim 5.73)$ & 0.135 \\
Molecular profile & 2.47 & $(0.93 \sim 6.59)$ & 0.071 \\
Surgery & 2.57 & $(1.00 \sim 6.62)$ & 0.051 \\
Chemotherapy & 2.90 & $(1.11 \sim 7.60)$ & 0.030 \\
Radiotherapy & 2.71 & $(1.05 \sim 7.01)$ & 0.040 \\
Endocrinetherapy & 2.18 & $(0.79 \sim 6.04)$ & 0.134 \\
Herceptin & (B) & $(0.98 \sim 6.54)$ & 0.055 \\
\hline
\end{tabular}

*obtained from the Cox model of proportional hazards adjusted for each variable;

$* *$ confidence interval;

$* * *$ statistical significance;

Source: The author, 2016.

Table 4 Multivariate Analysis of SIRT-1 adjusted for the occurrence of death according to the grouped prognostic variables in specific models in the cohort of female patients with breast cancer.

\begin{tabular}{llll}
\hline Models & HR* & IC 95\%** & $p^{* * *}$ \\
\hline SIRT-1 unadjusted & 2.66 & $(1.03 \sim 6.86)$ & 0.043 \\
SIRT-1, age & 2.86 & $(1.11 \sim 7.38)$ & 0.030 \\
SIRT-1, age, lymph nodes & 3.58 & $(1.35 \sim 9.52)$ & 0.011 \\
SIRT-1, age, TNM, histological grade & 2.27 & $(0.84 \sim 6.15)$ & 0.108 \\
SIRT-1, age, lymph nodes, Her-2, Ki-67 & 3.82 & $(1.37 \sim 10.64)$ & 0.010 \\
SIRT-1, age, lymph nodes, hormone receptor & 2.87 & $(0.97 \sim 8.48)$ & 0.056 \\
SIRT-1, age, lymph nodes, molecular profile & 3.44 & $(1.21 \sim 9.79)$ & 0.021 \\
\hline
\end{tabular}

*obtained from the Cox model of proportional hazards adjusted for each variable;

$* *$ confidence interval;

$* * *$ statistical significance;

Source: The author, 2016. 
In this regard, several studies seek to identify molecular markers that independent or associated with existing markers can identify subgroups of patients for more effective and selective treatments. Some surveyed markers, such as cyclin D1, cyclin E, DBC1, INK4a/ARF, TXB2/3 and VEGF express prognostic differences among subgroups of tumors, but are not consolidated in clinical practice [24, 25].

Given the complexity and heterogeneity of breast cancer biology, there is a need to investigate the prognostic effect of new molecules, such as sirtuin-1 (SIRT-1), where there is evidence that changes in their expression, independent or associated the other surveyed variables may affect the natural history of the disease.

Sirtuins are a family of seven proteins (SIRT-1-7) identified in mammals, classified as histone Class III, because they depend on the NAD+ (nicotinamide adenine dinucleotide) to perform their activities in the deacetylation and mediation of various cellular metabolic functions [26-29]. Sirtuin-1 (SIRT-1) is a kind of labile molecules circulating in the nuclear, cytoplasmic and membrane environments, exerting positive or negative action in signaling actions with various molecules and substrates important in epigenetic regulation [25, 26, 29]. Biologically, in humans, SIRT-1 is related to many critical processes of cells, among these, the genomic stability, cell proliferation, resistance, response to stress and cell aging [27, 29, 30]. In this context, several studies correlated with SIRT-1 stages of initiation and cell proliferation in oncogenesis, but in some models, the SIRT-1 operates with suppressive function and in other as a cancer promoter function. Given the complexity of factors involved in tumorigenesis, it remains controversial the function of SIRT-1 in these models [31].

Epigenetic changes, including histone modifications, are critical for mammary carcinogenesis, but the association of expression of SIRT1 with the clinical features and prognosis of breast cancer have not yet been clarified [32]. Changes in the level of expression of sirtuin-1 (SIRT-1) and other molecules related to the signaling pathways of tumor cells have been investigated in many types of tumors, including breast cancer, but the association degree these expressions with the clinical prognosis of patients have not been definitively determined [25, 33, 34].

In our research, we investigated the expression of SIRT-1 by immunohistochemistry in 123 cases of patients with breast cancer, which accounted for $26.9 \%$ of cases in the Erechim-RS cohort. According to the methodological criteria established for the investigation, we identified change in SIRT-1 expression in 114 patients (92.7\%), and 9 patients (7.3\%) showed no expression. Patients with changes in the grade of nuclear expression of SIRT-1 were investigated and observed, $71(57.7 \%)$ patients with low expression, 35 cases $(28.5 \%)$ with moderate expression and 8 cases $(6.5 \%)$ overexpressing the SIRT-1.

These findings confirm the hypothesis that there is a change in the expression of SIRT-1 in patients with breast cancer, but in varying grades of expression. Even though the laboratory techniques for the investigation of SIRT-1 expression are not uniform and there is lack of research in this area, the data found in the Erechim-RS research are consistent with the information in the literature that suggests that the change in SIRT-1 expression is associated with breast cancer [32].

In order to investigate the estimates of survival and the risk of death from breast cancer, according to the grade of SIRT-1 expression and its association with other variables studied in the Erechim cohort, patients were classified into two groups. The group classified as SIRT-1 negative was composed of patients with breast cancer who did not express change of SIRT-1 and patients with expression of SIRT-1 low or moderate, totalizing 115 patients (93.5\%). The group classified as SIRT-1 overexpressed was composed of eight patients who expressed high degree of SIRT-1 (6.50\%).To 
analyze the outcome of interest, during the cohort follow-up period, it was observed 30 deaths (26.1\%) in negative SIRT-1 group and five deaths (62.5\%)in overexpression SIRT-1 group. The survival median estimates calculated for the outcome of death from breast cancer during follow-up of the Erechim cohort, showed lower overall survival statistically significant for the overexpression SIRT-1 group patients. The risks related to the outcome of interest in the survey were calculated by univariate model (Cox).

In univariate analysis unadjusted, we found a relative risk (HR) of 2.66 times greater of death for patients who had SIRT-1 overexpression in relation to the group of patients without overexpression of SIRT-1. Through the univariate Cox model were calculated SIRT-1 effect on the risk of death from breast cancer, and adjusted for other variables studied and observed that the SIRT-1 maintained the association of increased risk of death when adjusted for age (HR 2.86 ; CI 95\% 1.11 7.38; $p=0.030$ ); histology (HR 2.79, CI 95\% $1.07 \sim 7.28 ; p=0.036)$; status of the axillary lymph nodes (HR 2.73, CI 95\% 1.06 7.04; $p=0.037$ ); Her-2 expression (HR 2.82, CI 95\% 1.07 7.44; $p=0.036$ ); completion of chemotherapy (HR 2.90, CI 95\% $1.11 \sim 7.60 ; p=0.030)$ and conducting radiation treatment (HR 2.71, CI 95\% 1.05 7.01; $p=0.040$ ). The data demonstrated an association with poor prognosis and a higher risk of death for patients who had SIRT-1 overexpression and higher age, SIRT-1 overexpression and metastatic involvement of axillary lymph nodes and overexpression of SIRT-1 and overexpression of Her-2.

The results of the univariate analysis showed increased risk of death for the association of SIRT-1 expression and the completion of chemotherapy or radiation therapy should be interpreted with caution. However, it suggests that patients with SIRT-1 overexpression have mechanisms of tumor resistance to systemic chemotherapy, as suggested in some studies [30, 31, 35].

The Cox multivariate model was constructed in order to test and verify the statistical significance of prognostic information obtained in the univariate model. In multivariate regression scenario, the variable grade of SIRT-1 expression was set and controlled according to traditional prognostic variables in breast cancer, and which had presented significance in the univariate model, such as age, the status of the lymph nodes, classification TNM, the histological grade of the tumor, the expression of hormone receptors, Her-2, Ki-67 index and the molecular profile. Consistently and significantly we observed that the patients classified with SIRT-1 overexpression showed lower survival and higher risk of death than patients classified with the absence of overexpression in several multivariate model scenarios created to control the effect of the degree of expression the SIRT-1, in the prognosis of patients with breast cancer.

From the information obtained in these analyzes, it was evident an estimate of poor prognosis and increased risk of death for patients with overexpression of SIRT-1, independent of the age of diagnosis of metastatic involvement of axillary lymph nodes and the molecular cancer profile breast cancer.

The results obtained in this study are relevant and suggest that patients with breast cancer with SIRT-1 overexpression have a more aggressive biological profile, independent from analysis of other factors or classical prognostic variables. Some evidences in the literature suggest that an important factor in SIRT-1 regulation is the $\mathrm{p} 53$ protein. These proteins could present interactions and be involved in its own feedback loop and, on balance, rise to a change in expression of one or both [13].

A recent study investigated a specific population, evaluated the serum levels of SIRT-1 and changes in gene polymorphism coding for the SIRT-1 in patients with breast cancer and found a significant association between higher serum levels of SIRT-1 gene polymorphism and prognosis in breast cancer [32].

The Asian meta-analysis, published in 2015, was the first study investigating the correlation of SIRT-1 
expression with classical prognostic factors, such as the TNM staging (AJCC) and status of axillary lymph nodes on survival of patients with breast cancer. In this study, we included six trials totaling 604 patients and it was found a significant association between changes in SIRT-1 expression and lower survival from breast cancer. In the analyzes we investigated the expression of SIRT-1, TNM staging (AJCC) and the status of the axillary lymph nodes, having been a significant association between increased expression of SIRT-1, more advanced clinical stages and metastatic involvement of lymph nodes axillary [34].

Some recent research in breast carcinogenesis suggest theoretical models considering a complex interaction of genetic and epigenetic mechanisms involved in the processes of initiation and development of cancer. Investigations assume that epigenetic changes may be reversible and be related to changes in the expression of proteins and molecules that regulate the metabolism of histones, DNA methylation and mRNA expression levels [36]. These evidences indicate the complexity of the breast oncogenesis and the possibility that changes in the expression of SIRT-1 may be involved in the characterization of a particular subgroup of breast cancer.

To understand the effect of overexpression of SIRT-1 in breast cancer, and explain the clinical outcome of poor prognosis, based on literature review and findings, we propose a synthesis of the hypothetical theoretical model. Tumor tissue is characterized by fluctuations in the oxygen concentration, reduced nutrients supply and acidic $\mathrm{pH}$, with increased glucose consumption and lactate secretion increases [37]. Overexpression of SIRT-1 in the cell nucleus would cause a metabolic imbalance, cellular stress, the activation flags angiogenesis pathways and changes in pathway expression PI3K (phosphatidylinositol 3 protein kinase), which are related to tyrosine kinase receptors, thus causing an increase of cell proliferation and apoptosis loss of capacity [38]. The hyperactivation of SIRT-1 molecules alter the expression of $\mathrm{p} 53$ protein (transcription factor that regulates the cell cycle) resulting in dysregulation of signaling pathways of steroid receptors $[19,39]$. This intracellular homeostatic imbalance would lead to an inhibition of ligands related to the transcription of hormone receptors, capable of changing phenotypes in breast cancer [39]. This altered cellular environment, becomes favorable to the development of resistance of tumors to therapy employed and progression of tumor cells and thereby expressing a tumor molecular profile with more aggressive biological behavior and poor clinical outcome for patients.

\section{Final Considerations}

The evaluation of SIRT-1 expression by immunohistochemistry technique in tumor tissue from paraffin blocks proved feasible but requires a standardization of the technique aiming at standardization of information and the possibility of reproducibility and comparison of this information by other research centers. The findings of this research have shown that SIRT-1 was the independent prognostic marker of risk of death for patients with breast cancer. Patients with SIRT-1 overexpression featured a special subgroup of breast cancer that had a poorer prognosis.

In this research, SIRT-1 showed evidence that qualify it as a "new biomarker" relevant in breast cancer setting. Thus, further studies are needed and future prospective investigations and in larger series to confirm these findings and to understand the true impact of overexpression of SIRT-1 in the prognosis of breast cancer. Moreover, new studies will allow the development of a rational model of bio-molecular action of the SIRT-1 allowing in the future, the development of specific target drugs anti-SIRT-1.

\section{Conclusions}

This study allows us to conclude that, in the studied cohort: 
- The median estimates of overall survival for breast cancer in 5 and 10 years were $79.6 \%$ and $69.1 \%$, respectively;

- Patients classified overexpressing sirtuin-1 (SIRT-1) characterized a subgroup of women who were strongly associated with poor prognosis, shorter survival and increased risk of death in breast cancer, independently from the adjustment for age, status axillary lymph nodes, Her-2 expression and proliferation index (Ki-67);

- The grade of expression of SIRT1 in tumor tissue may be an important prognostic marker in risk assessment for women with breast cancer.

\section{Conflict of Interest}

There is no potential conflict of interest.

\section{References}

[1] Polyak, K. 2007. "Breast Cancer: Origins and Evolution." J. Clin. Invest. 117: 3155-63.

[2] Shiovitz, S., and Korde, L. A. 2015. "Genetics of Breast Cancer: A Topic in Evolution." Annals of Oncology 26: 1291-9.

[3] Hortobagyi, G. N., El-Saghir, N. S., Cufer, T., Cazap, E., De Guzman, R., Othieno-Abinya, N. A., Sanchez, J. A., et al. 2016. "The American Society of Clinical Oncology's Efforts to Support Global Cancer Medicine." J. Clini. Oncol. 34 (1): 76-82,

[4] Siege, R. L., Miller, K. D., and Jemal, A. 2016. "Cancer Statistics, 2016." CA Cancer J. Clin. 66 (1): 7-30. doi: 10.3322/caac.21332.

[5] Ministry of Health, National Secretariat of Health Care, National Cancer Institute. 2016. Coordination of Prevention and Surveillance. Estimate 2016: Cancer Incidence in Brazil. Rio de Janeiro: INCA.

[6] Sotiriou, C., Phil, D., and Puzstai, L. 2009. "Gene-Expression Signatures in Breast Cancer." N. Engl. J. Med. 360: 790-800. Accessed February 19, 2009. www.nejm.org.

[7] Cancer Genome Atlas Network. 2012. "Comprehensive Molecular Portraits of Human Breast Tumors." Nature 490: 61-70. doi: 10.1038/nature11412.

[8] Carvalho, F. M. 2007. "Histopathology." In Breast Cancer: A Multidisciplinary Treatment, edited by Barros, A. C. S. D., and Buzaid, A. C. São Paulo: Dendrix, 3-25.

[9] Senkus, E., Kyriakides, S., Ohno, S., Penault-Llorca, F., Poortmans, P., Rutgers, E., et al. 2015. "On Behalf of the ESMO Guidelines Committee. Primary Breast Cancer:
ESMO Clinical Practice Guidelines for Diagnosis, Treatment and Follow-Up." Annals of Oncology 26 (Supplement 5): v-30. doi: 10.1093/annonc/mdv298. Accessed $\quad$ February 2016. http://annonc.oxfordjournals.org.

[10] Tan, D. S. W., Mok, T. S. K., and Rebbeck, T. R. 2016. "Cancer Genomics: Diversity and Disparity across Ethnicity and Geography.” J. Clin. Oncol. 34 (1): 91-101,

[11] Locke, W. J., and Clark, S. J. 2012. "Epigenome Remodeling in Breast Cancer: Insights from an Early Model of Carcinogenesis." Breast Cancer Research 14: 215. doi: 10.1186/bcr3237.

[12] Blander, G., and Guarente, L. 2004. "The Sir2 Family of Protein de Acetylases.” Annual Review of Biochemistry 73: 417-35.

[13] Presegué-Bosch, L., and Vaquero, A. 2011. "The Dual Role of Sirtuins in Cancer." Genes \& Cancer. doi: $10.1177 / 1947601911417862$.

[14] Hall et al. The sirtuin family's role in aging and age-associated pathologies. J Clin Invest. 2013; v.123, n.3, p.973-979. doi:10.1172/JCI64094.

[15] Dali-Youcef, N., Lagouge, M., Froelich, S., Koehl, C., Schoonjans, K., and Auwerx, J. 2007. "Sirtuins: The "Magnificente Seven", Function, Metabolism and Longevity." Ann. Med. 39: 335-45. doi: 10.1080/07853890701408194. PMID: 17701476.

[16] Chackrabarty, S. P., Balaram, H., and Chandrasekaran, S. 2011. "Sirtuins: Multifaceted Drug Targets." Current Molecular Medicine 11 (9): 1875-5666.

[17] Mellini, P., Valente, S., and Mai, A. 2015. "Sirtuin Modulators: An Updated Patent Review (2012-2014)." Expert Opin. Ther Pat. 25 (1): 5-15. doi: 10.1517/13543776.2014.982532.

[18] Hwang, B. J., Madabushi, A., Jin, J., Lin, S. Y., and Lu, A. L. 2014. "Histone/Protein Deacetylase SIRT1 is an Anticancer Therapeutic Target.” Am. J. Cancer Res. 4 (3): 211-21.

[19] Elangovan, S., Ramachandran, S., Venkatesan, N., Ananth, S., Gnana-Prakasam, J. P., Martin, P. M., et al. 2011. "SIRT1 Is Essential for Oncogenic Sgnaling by Estrogen/Estrogen Receptor $\alpha$ in Breast Cancer." Cancer Res. 71 (21): 6654-64.

[20] Mitchell, H. K. 2003. "Multivariable Analysis: A Primer for Readers of Medical Research.” Ann Intern Med. 138: 644-50.

[21] Perou, C. M., Sørlie, T., Eisen, M. B., Van de Rijn, M., Jeffrey, S. S., Rees, C. A., et al. 2000. "Molecular Portraits of Human Breast Tumours." Nature 406: 747-52.

[22] Nik-Zainal, S., Davies, H., Staaf, J., Ramakrishna, M., Glodzik, D., Zou, X., et al. 2016. "Landscape of Somatic Mutations in 560 Breast Cancer Whole-Genome Sequences." Nature 534 (7605): 47-54. doi: 
10.1038/nature17676

[23] Inoue, K., and Fry, E. A. 2016. "Novel Molecular Markers for Breast Cancer." Biomark Cancer 8: 25-42. doi: 10.4137/BIC.S38394.

[24] Taneja, P., Maglic, D., Kai, F., Zhu, S., Kendig, R. D., Fry, E. A., et al. 2010. "Classical and Novel Prognostic Markers for Breast Cancer and their Clinical Significance." Clinical Medicine Insights: Oncology 4: 15-34.

[25] Lee, H., Kim, K. R., Noh, S. J., Park, H. S., Kwon, K. S., Park, B. H., et al. 2011. "Expression of DBC1 and SIRT1 is Associated with Poor Prognosis for Breast Carcinoma." Hum. Pathol. 42: 204-13.

[26] Cantó, C., Gerhart-Hines, Z., Feige, J. N., Lagouge, M., Noriega, L., Milne, J. C., et al. 2009. “AMPK Regulates Energy Expenditure by Modulating NAD+ Metabolism and SIRT1 Activity." Nature 458 (7241): 1056-60.

[27] Finkel, T., Deng, C. X., and Mostoslavsky, R. 2009. "Recent Progress in the Biology and Physiology of Sirtuins." Nature 460 (7255): 587-91. doi:10.1038/nature08197.

[28] Wierman, M. B., and Smith, J. S. 2014. "Yeast Sirtuins and the Regulation of Aging." FEMS Yeast Res. 14 (1): 73-88. doi:10.1111/1567-1364.12115.

[29] Li, S., Hong, H., Lv, H., Wu, G., and Wang, Z. 2016. "SIRT 1 Promotes Migration and Growth of PDAC Cells." Med. Sci. Monit. 22: 1593-600.

[30] Cao, B., Shi, Q., and Wang, W. 2015. "Higher Expression of SIRT1 Induced Resistance of Esophageal Squamous Cell Carcinoma Cells to Cisplatin.” J. Thorac. Dis. 7 (4): 711-9. doi: 10.3978/j.issn.2072-1439.2015.04.01. PMCID: PMC4419326.

[31] Li, K., and Luo, J. 2011. "The Role of SIRT1 in Tumorigenesis.” N. Am. J. Med. Sci. (Boston) 4 (2): 104-6.

[32] Rizk, S. M., Shahin, N. N., and Shaker, O. G. 2016. "Association between SIRT1 Gene Polymorphisms and
Breast Cancer in Egyptians.” PLoS ONE 11 (3): e0151901. doi:10.1371/journal.pone.0151901.

[33] Cao, Y. W., Li, W. Q., Wan, G. X., Li, Y. X., Du, X. M., Li, Y. C., et al. 2014. "Correlation and Prognostic Value of SIRT1 and Notch1 Signaling in Breast Cancer." J. Exp. Clin. Cancer Res. 33: 97. doi: 10.1186/s13046-014-0097-2.

[34] Cao Y. W., Li, Y. C., Wan, G. X., Du, X. M., and Li, F.. 2015. "Clinicopathological and prognostic role of SIRT1 in breast cancer Patients: a meta-analysis." Int. J. Clin. Med. 8 (1): 616-24. Accessed January 20, 2016. www.ijcem.com.

[35] Chen, H. C., Jeng, Y. M., Yuan, R. H., Hsu, H. C., and Chen, Y. L. 2012. "SIRT1 Promotes Tumorigenesis and Resistance to Chemotherapy in Hepatocellular Carcinoma and Its Expression Predicts Poor Prognosis." Ann. Surg. Oncol. 19: 2011-9. doi: 10.1245/s10434-011-2159-4 PMID: 22146883.

[36] Byler, S., Goldgar, S., Heerboth, S., Leary, M., Housman, G., Moulton, K., et al. 2014. "Genetic and Epigenetic Aspects of Breast Cancer Progression and Therapy." Anticancer Res. 34: 1071-7. doi: 34/3/1071. PMID: 24596345.

[37] Harjes, U., Bensaad, K., Harris, A. L. 2012. "Endothelial Cell Metabolism and Implications for Cancer Therapy." Br. J. Cancer 107 (8): 1207-12. doi: 10.1038/bjc.2012.398.

[38] Byles, V., Chmilewski, L. K., Wang, J., Zhu, L., Forman, L. W., Faller, D. V., et al. 2010. "Aberrant Cytoplasm Localization and Protein Stability of SIRT1 is Regulated by PI3K/IGF-1R Signaling in Human Cancer Cells.” Int. J. BiolSci. 6 (6): 599-612.

[39] Moore, R. L., Dai, Y., and Faller, D. V. 2012. "Sirtuin1 (SIRT1) and Steroid Hormone Receptor Activity in Cancer." Journal of Endrocrinology 213 (1): 37-48. 